\title{
Diseño de una red de acceso de fibra óptica para proveer video, internet y evaluación de parámetros de calidad sobre la red caso de estudio: Huambaló
}

DOI: https://doi.org/10.33262/ap.v3i3.1.88

\begin{abstract}
(c) (1) (2)
Design of a Fiber Optic Access Network to Provide Video and Internet and Evaluation of quality parameters on the network case study "Huambalo Sector"
\end{abstract}

Oswaldo Martínez Guashima. ${ }^{1}$, Oscar Sebastián Parra Mayorga. ${ }^{2}$ \& Irvin André Peñafiel Ortega. ${ }^{3}$

\begin{abstract}
.
Introduction. this study was to design a fiber-optic access network to provide video and data services, test the operation of the network in the Huambaló sector. With the maps of the sector, a network was designed adapted to the standards of Optical Network with Gigabit Capacity (GPON). Methodology. Advantages and disadvantages with the current network where a map of a converging optical network with single-mode fiber was designed. Using the OptiSystem software, Results. the network was simulated with the two most critical closest and farthest user of each of the zones, testing the technical transmission parameters: attenuation, bit error rate (BER), and quality factor Q. Conclution. as a result, the values of both cases are within the ITU G.984.2 class C+

Keywords: Telecommunications, Optical Network with Gigabit Capacity

\footnotetext{
${ }^{1}$ Escuela Superior Politécnica de Chimborazo, Facultad de Informática y Electrónica. Riobamba, Ecuador. omartinez@espoch.edu.ec. https://orcid.org/0000-0001-9018-7777

${ }^{2}$ Escuela Superior Politécnica de Chimborazo, Facultad de Informática y Electrónica. Riobamba, Ecuador. oscar.parra@espoch.edu.ec. https://orcid.org/0000-0002-1034-2544

${ }^{3}$ Instituto Tecnológico Superior La Mana, Carrera de Redes y Telecomunicaciones. La Mana Ecuador. ipenafiel@istlamana.edu.ec. https://orcid.org/0000-0002-4637-1005
} 


\section{Resumen.}

Objetivo Este estudio fue diseñar una red de acceso de fibra óptica para proveer los servicios de video y datos, evaluar el funcionamiento de la red en el sector de Huambaló. Con los planos del sector se diseñó una red que se adecue a los estándares de Red Óptica con Capacidad Gigabit (GPON), Metodologia. Donde se analizó ventajas y desventajas con la red actual donde se diseñó un plano de una red óptica convergente con fibra de tipo monomodo, mediante la utilización del software de OptiSystem Resultados. Se simuló la red con los dos casos más críticos usuario más cercano y lejano de cada una de las zonas, evaluando los parámetros técnicos de transmisión: atenuación, tasa de error de bits (BER) y factor de calidad Q. Conclusion, Los valores de los dos casos se encuentran dentro de la recomendación ITU G.984.2 clase C+

Palabras claves: Telecomunicaciones, Red Óptica con Capacidad de Gigabit.

\section{Introducción.}

El acceso a los servicios de televisión pagada e internet con mejores prestaciones como una taza alta de transferencia de datos es un problema que se presenta en las zonas rurales en la actualidad. Existen diferentes proyectos técnicos que dan solución a este problema desde el aumento de ancho de banda hasta soluciones más efectivas como el cambio de infraestructura de la red de cobre a fibra óptica.

Debido al crecimiento comercial de las industrias y la tecnología GPON (Red Óptica Pasiva con Capacidad de Gigabit) las empresas buscan expandir sus servicios como televisión e internet a través de un mismo medio de transmisión. Por lo que la fibra óptica permite utilizar una sola infraestructura de acceso reduciendo el consumo de recursos.

En 1820, Augustine-Jane Fresnes ya sabía de las leyes matemáticas que presidian sobre la recepción de luz en muestras de vidrio. En el año 1870 el físico John Tyndall que provenía del país de Irlanda encontró lo efectos que se presentaba la luz como la reflexión interior que se da en el agua al momento que la misma viaja a través de ella. Desde entonces se seguían presentando estudios donde exponían acerca del potencial que tenía el vidrio para transportar la luz a largas distancias de una manera eficiente.

La empresa American Cable brinda el servicio de televisión por suscripción en la ciudad de Pelileo con una red de cobre y planea hacer una migración de su tecnología hacia una nueva.

En investigaciones se conoció que existe un estudio previo no perteneciente a la empresa realizados por terceros de un diseño de red GPON para la ciudad de Pelileo es por eso que se ha tomado a la parroquia de Huambaló como base, desde el punto de vista técnico donde se ha planteado un análisis desde un enfoque a los servicios de telecomunicaciones con mayor demanda como son internet y televisión, por su importancia de manera local 
como global debido que hoy en día la mayoría de actividades se realizan de una manera online y el entretenimiento digital va en aumento.

Es por eso por lo que se desea transmitir estos servicios en las ventanas de transmisión que presentan menor atenuación de la tecnología de fibra óptica donde se ha utilizado una normativa diferente que se adapte a las necesidades de los servicios y de la parroquia donde se va a realizar el diseño de red.

La parroquia rural Huambaló donde habitan alrededor de 16000 habitantes de los cuales 11200 se dedican a la fabricación y comercialización de muebles finos siendo así su principal actividad económica. Donde actualmente acceden al servicio de internet por el medio de transmisión de cobre, donde se alcanzan velocidades de transmisión de hasta $15 \mathrm{Mbps}$ en un plan elevado que ofrece CNT E.P. en la zona.

Debido al crecimiento exponencial de las tecnologías y de los servicios que hoy en día se brinda por internet muchos de los comerciantes están siguiendo la tendencia de las ventas en línea necesitando así un gran ancho de banda para cubrir todas las necesidades que exige realizar dichas ventas a través de una plataforma virtual.

\section{Marco teórico}

\section{A. Normativa ITU-T G.984.x}

Es desarrollado y estandarizado por la ITU-T (Internacional Telecomunication Union) para la tecnología GPON, que cubre desde los servicios de trasporte TDM, como información datos y video, utilizando tramas ATM o a su vez Ethernet llegando a una velocidad de $1.25 \mathrm{Gbps}$ en el canal upstream y $2.5 \mathrm{Gbps}$ en el canal downstream. Se establecen ciclos de transmisión definidos con un tiempo en $125 \mu \mathrm{s}$, donde se fijaron mecanismos de fragmentación cada una de las tramas ethernet para las ONT, así como para el OLT, que serán trasmitidos en los slots de tiempos ya preestablecidos por el OLT. Para el correcto funcionamiento de toda la red PON se necesitan ciclos fijos de sincronización.

Tabla 1

CLASES DE LA TECNOLOGÍA GPON

\begin{tabular}{cccc}
\hline Clases & Estándar ITU & $\begin{array}{c}\text { Perdidas } \\
\text { mínimas }\end{array}$ & $\begin{array}{c}\text { Perdidas } \\
\text { máximas }\end{array}$ \\
\hline$A$ & G.984.2 / G.982 & $5 \mathrm{~dB}$ & $20 \mathrm{~dB}$ \\
$B$ & G.984.2 / G.982 & $10 \mathrm{~dB}$ & $25 \mathrm{~dB}$ \\
$B+$ & G.984.2 enmienda 1 (2006) & $13 \mathrm{~dB}$ & $28 \mathrm{~dB}$ \\
$C$ & G.984.2 / G.982 & $15 \mathrm{~dB}$ & $30 \mathrm{~dB}$ \\
$C+$ & G.984.2 enmienda 2(2008) & $17 \mathrm{~dB}$ & $32 \mathrm{~dB}$ \\
\hline
\end{tabular}

Fuente: TELNET (2014)

De lo que se muestra en la tabla I anterior existe un amplio catálogo de equipos y de elementos activos que manejan esos niveles de perdidas mínimas y máximas. 
Donde los equipos que se utilizan para la transmisión y recepción la recomendación ITU G.984.2 clase $C+$ se da los parámetros de potencia máxima de transmisión de $+7 \mathrm{dBm}$ en la OLT y $+3 \mathrm{dBm}$ potencia mínima de transmisión en la OLT, una sensibilidad mínima en la ONT de -30 dBm y una sobrecarga mínima $-8 \mathrm{dBm}$ en la ONT. Siendo este estándar el más optimo que se utilizara para el diseño de la red ya que se tiene un margen mayor de potencia mínima y máxima en respecto a las demás clases.

Tabla 2

NIVELES DE POTENCIAS ÓPTICOS DE CLASE C+.

\begin{tabular}{|l|c|c|}
\hline \multicolumn{1}{|c|}{ Item } & Unit & Single fibre \\
\hline Reach-extended OLT: & & OLT \\
\hline Mean launched power MIN & $\mathrm{dBm}$ & +3 \\
\hline Mean launched power MAX & $\mathrm{dBm}$ & +7 \\
\hline Downstream optical penalty & $\mathrm{dB}$ & 1 \\
\hline Bit error ratio (pre-FEC) [Note 1] & & $10^{-4}$ \\
\hline Minimum sensitivity [Note I] & $\mathrm{dBm}$ & -32 \\
\hline Minimum overload & $\mathrm{dBm}$ & -12 \\
\hline $\begin{array}{l}\text { Upstream wavelength range } \\
\text { [ITU-T G.984.5] }\end{array}$ & $\mathrm{nm}$ & $1290-1330$ \\
\hline ONU: & - & $0 \mathrm{NU}$ \\
\hline Mean launched power MIN & $\mathrm{dBm}$ & +0.5 \\
\hline Mean launched power MAX & $\mathrm{dBm}$ & +5 \\
\hline Upstream optical penalty & $\mathrm{dB}$ & 0.5 \\
\hline $\begin{array}{l}\text { Upstream wavelength range } \\
\text { [ITU-T G.984.5] }\end{array}$ & $\mathrm{nm}$ & $1290-1330$ \\
\hline Bit error ratio (pre-FEC) [Note 2] & - & $10^{-4}$ \\
\hline Minimum sensitivity [Note 2] & $\mathrm{dBm}$ & -30 \\
\hline Minimum overload [Note 3] & $\mathrm{dBm}$ & -8 \\
\hline
\end{tabular}

FUENTE: ITU G.984.2, (2019)

\section{METODOLOGÍA}

\section{B. Diseño}

Se realizó una investigación no experimental, debido a que los equipos no se pueden implementar en esta investigación por su elevado costo de adquisición, por ser de una estructura muy extensa debido a al factor demográfico que presenta el sector al ser una zona rural de 3 kilómetros de radio aproximadamente y con un relieve bastante irregular.

Se propone una innovación a la tecnología existente de cobre remplazándola con fibra óptica tipo monomodo ya que presta mejores características y utilizando escenarios de pruebas mediante software de simulación. Es un diseño transversal debido a que se realiza el análisis, la incidencia e interrelación de la tecnología actual a la nueva que se propone.

\section{Enfoque}

Se tiene un enfoque cuantitativo ya que se tomó como punto de partida algo que no fue mesurable, es decir que está relacionada con los planos de la zona referentemente al 
acceso a servicios de telecomunicaciones y después se pasara a algo que se pueda cuantificar, en base a entrevistas en la zona, cuantas personas acceden a estos servicios, en otras palabras son datos que al término de esta investigación aportaran conclusiones.

\section{Localización}

El sector de Huambaló perteneciente al cantón de Pelileo de la provincia de Tungurahua, está situado a $7 \mathrm{Km}$ de la ciudad antes mencionada con una extensión de $25.87 \mathrm{~km} \wedge 2 \mathrm{y}$ posee una población estimada de 10034 habitantes. Para este estudio se tomó en cuenta el número viviendas por sector debido a que los servicios de telecomunicaciones son contratados un contrato por todos los integrantes de la familia y por el sector económicamente más activa donde se ha dividido en 8 sectores principales.

- Huambaló centro

- La Florida

- $\quad$ La Merced

- San Antonio

- San Francisco

- San José

- Segovia

- $\quad$ Surangay

Donde se evidencia que 7 de los 8 sectores presentan se encuentran económicamente activo, dejando solo al sector de San Antonio en una actividad más baja en relación con la demás.

\section{Gráfico 1}

Sectores de activa económica

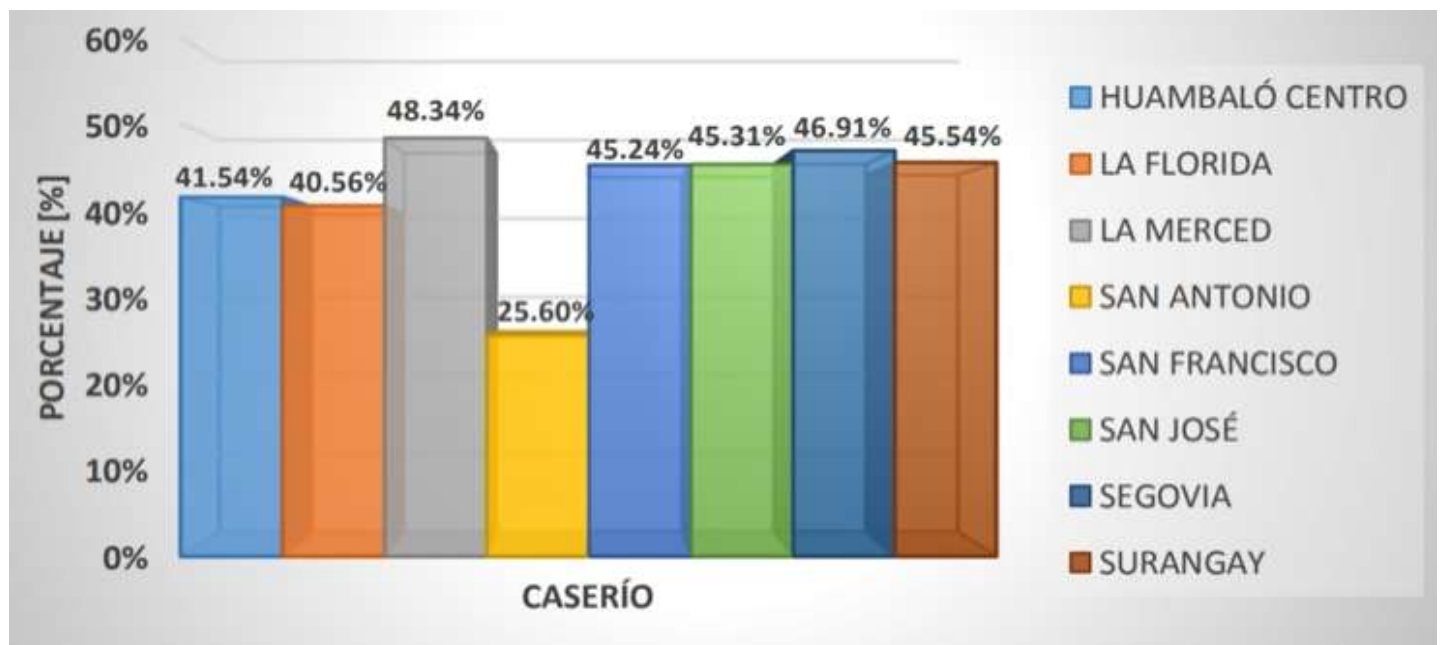

Fuente: Plan de desarrollo y ordenamiento territorial de la parroquia rural Huambaló. (Pavón and GADPR Huambaló, 2015)

Alrededor de 921 personas se dedican a la industria de manufactura de muebles finos de alta calidad siendo la segunda activada económica más importante de la parroquia solo por detrás de la agricultura ganadería, silvicultura y pesca. Donde existen alrededor de 
40 talleres dedicados a la fabricación y 80 locales comerciales dedicados a la venta de los mismo.

Como establecimientos importantes en el sector de Huambaló se tiene ubicados en su mayoría en la zona céntrica de la parroquia.

- 7 unidades educativas

- centros infantiles del buen vivir

- 1 subcentro de salud

- 1 dispensario, medico campesino del IESS

- $\quad$ sucursales de cooperativas de ahorro y crédito

- restaurantes

- centros de cómputo.

\section{E. Estudio de demanda}

En la elaboración del diseño, se partió con encuestas en el sector de estudio para establecer una población inicial, las necesidades que tienen los clientes finales ya sea en sector residencial como el sector industrial donde ellos serán los usuarios los que se pueda brindar los servicios de telecomunicaciones y en base a ello establecer la tecnología y elementos para la red para cada uno de ellos.

En el análisis urbanístico del sector en el diseño un factor a tomar en cuenta es identificar el número de viviendas que cuenta el sector, donde para calcular cuentas existen se realizó un conteo del número de casas construidas en la en base al mapa urbanístico de la parroquia proporcionado por el Gobierno Autónomo Descentralizado de Pelileo.

\section{Figura 1}

Mapa urbanístico de Huambaló

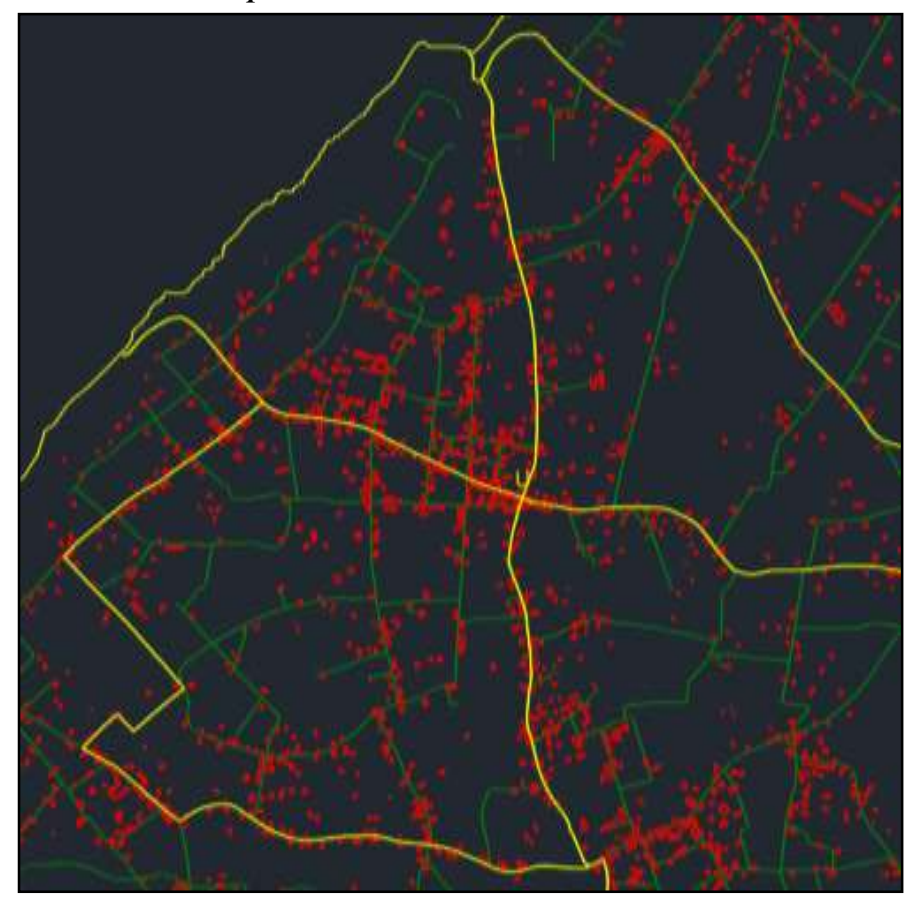

Fuente: Elaboración propia. 
Donde se contabilizase cada uno de los rectángulos rojos del mapa como resultado se obtuvo 2508 construcciones.

$$
\begin{gathered}
n=\frac{2508}{0.05^{2}(2508-1)+1} \\
n=345.09
\end{gathered}
$$

Entonces como resultado se tiene que se deben realizar 345 encuetas, cada encuesta se realizara a un integrante del hogar que correspondería a usuario final de la parroquia.

Después de que se estudió cada una de las respuestas del cuestionario se puede comprobar que el $60 \%$ de futuros clientes ha pensado en contratar el servicio de internet y el $27 \%$ en contratar ambos servicios televisión e internet como se observa en el grafico 1-3, de igual manera el $84 \%$ de las personas encuestadas están de acuerdo con un cambio de tecnología hacia fibra óptica.

\section{Gráfico 2}

Respuestas de las preguntas más relevantes de la encuesta realizada
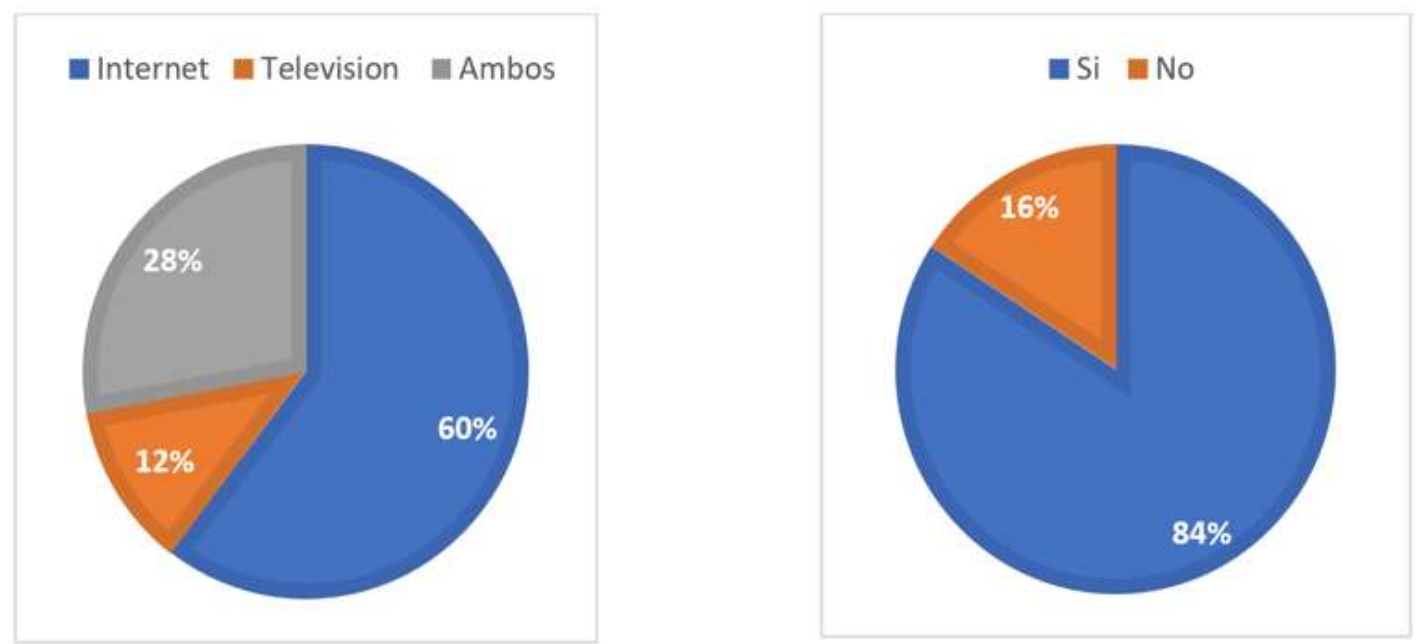

Fuente: Elaboración propia.

Posteriormente al análisis de las respuestas se puede evidenciar que es necesario un cambio de tecnología de cobre hacia fibra óptica ya que las encuestas realizadas aleatoriamente en la parroquia rural de Huambaló respaldan y aseveran que es aceptada la propuesta tecnología del trabajo de investigación.

\section{F. Propuesta tecnológica de diseño de la red}

Consiste en identificar los componentes que estarán presentes en la red y en la cual se aplicara la tecnología GPON, donde estará divida en dos partes principales: la parte del diseño lógico y el diseño físico

En el diseño lógico se toma en cuenta la delimitación de la parroquia Huambaló, su relieve, arquitectura, topología y los requerimientos velocidad para cada zona del usuario. 
Figura 2

Diseño de la red GPON

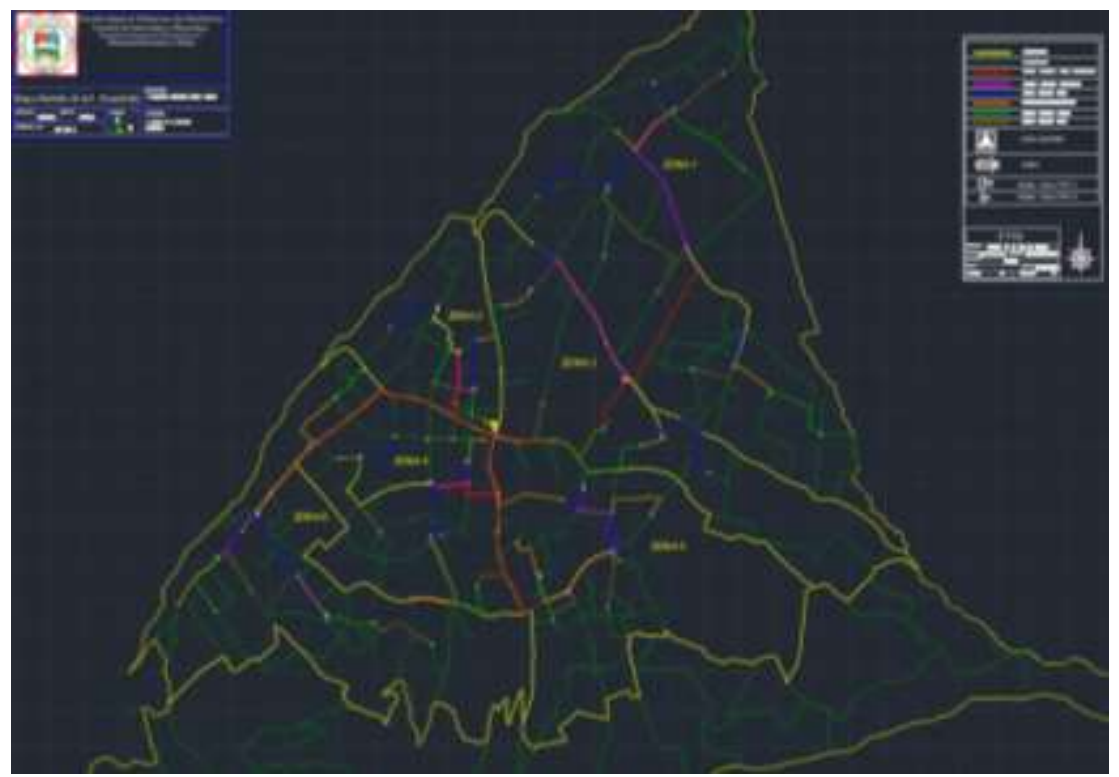

Fuente: Elaboración propia.

Diseño de la red GPON

Donde se realizó una división del mapa en 6 zonas específicas: 4 centrales que es donde se presenta mayor concentración demográfica, una en la parte norte y otra en la al sur para así tener un mayor control de los usuarios

Para el diseño de red se utilizó 3 red feeder o también conocidas como redes troncales. Es aquella que se conecta desde el armario de distribución óptica ubicado en la OLT hasta la maga de sangrado llamado así por qué se hace un corte en la fibra principal donde solo se toma solo un número de hilos necesarios para cubrir una determinada zona.

Red de distribución que es la encargada de distribuir todos los hilos asignados a la zona desde la maga hasta cada una de las NAP (Punto de acceso de red) que se encuentran en segundo nivel de splitter y con el primer nivel de splitter. Su función principal es de cubrir un sector específico de la zona, por lo general son ubicadas por una manzana o dos específicamente dependiendo de las dimensiones de cada una.

Red de dispersión esta última parte de la de la red, es la que llega hasta el usuario final desde el segundo nivel de splitter hasta cada una de las ONT de las viviendas de los futuros clientes.

\section{G. Recursos Materiales}

Se va a analizar cada uno de los materiales de la red, las características físicas y técnicas de los equipos que se propone utilizar para el diseño de la red, se ha separado en dos los elementos, activos y pasivos.

\section{OLT}


Es el elemento principal de la red, este debe contar con las características técnica especificadas por la normativa ITU-T G.984.2. clase $\mathrm{C}+$ para un correcto funcionamiento, además de propiedades fundamentales de escalabilidad de la red para cumplir con todos los usuarios potenciales y futuros, compatibilidad con los demás equipos finales de la red, seguridad, QoS (calidad de servicio) y control de usuarios.

Se ha tomado en cuenta dos modelos de equipos OLT que actualmente se encuentra en el mercado ecuatoriano para analizar cada uno de ellos y determinar cuál es el más adecuado para el diseño de la red.

\section{Tabla 3}

NIVELES DE POTENCIAS ÓPTICOS DE CLASE C+.

\begin{tabular}{lcc}
\hline Equipo & HUAWEI MA5680T & ZTE C300 \\
\hline Categoría GPON & Class B+ and Class C+ & Class B+ and Class C+ \\
\hline Longitud de onda operativa & Tx: $1550 \mathrm{~nm}$ Rx:1310 $\mathrm{nm}$ & Tx: $1550 \mathrm{~nm}$ Rx:1310 \\
\hline Tipo de encapsulación & SFP & SPF \\
\hline Tazas de velocidades & Tx: 2.49 Gbps Rx: 1.25Gbps & $\begin{array}{c}\text { Tx: } 2.49 \mathrm{Gbps} \text { Rx: } \\
1.25 \mathrm{Gbps}\end{array}$ \\
\hline $\begin{array}{l}\text { Potencia óptica de salida } \\
\text { mínima }\end{array}$ & $3 \mathrm{dBm}$ & $4.5 \mathrm{dBm}$ \\
\hline $\begin{array}{l}\text { Potencia óptica de salida } \\
\text { máxima }\end{array}$ & $7 \mathrm{dBm}$ & $8 \mathrm{dBm}$ \\
\hline $\begin{array}{l}\text { Sensibilidad máxima del } \\
\text { receptor }\end{array}$ & $-32 \mathrm{dBm}$ & $-32 \mathrm{dBm}$ \\
\hline Tipo de conector & SC/PC & SC/PC \\
\hline Fibra Óptica & Monomodo & Monomodo \\
\hline Relación de división & Min:16 Max:128 & Min: $16 \mathrm{Max}: 128$ \\
& Sugerida: 64 & Sugerida: 64 \\
\hline Distancia & $20 \mathrm{Km}$ & $20 \mathrm{Km}$ \\
\hline Precio & $\$ 18000.00$ & $\$ 19500.00$ \\
\hline
\end{tabular}

Fuente: (“Huawei MA5680T Price and Datasheet," 2020; "ZTE C300 Price And Datasheet," 2020)

\section{CARACTERÍSTICAS DE LOS DIFERENTES MODELOS DE OLT}

Se ha elegido el equipo HUAWEI MA5680T, desde el punto de vista de potencia ya que lo que se desea saber es comprobar uno de los casos críticos de la red del usuario más lejano, con la mínima potencia que se puede encontrar en el mercado para garantizar un correcto funcionamiento de la red y por su menor costo con respecto al equipo ZTE C300.

\section{H. Presupuesto comercial de los recursos materiales}

A continuación, se detallará los costos de cada uno de los equipos activos como pasivos que intervendrán en el diseño de la red GPON para conocer cuál sería la inversión de este proyecto, donde se especifica cada uno de los precios unitarios de los equipos y el precio 
total. Estos precios se han averiguado en varias empresas de telecomunicación que se dedican a la venta y distribución de equipos y material de fibra óptica.

Tabla 4

COSTO REFERENCIAL DE LA INVERSIÓN PARA LA RED GPON

\begin{tabular}{crrrrr}
\hline Equipos & Cantidad & \multicolumn{2}{c}{ Valor Unitario } & \multicolumn{1}{c}{ Valor Total } \\
\hline OLT & 1 & $\$$ & $18.000,00$ & $\$ 18.000,00$ \\
\hline ONT & 2048 & $\$$ & 54,99 & $\$ 112.619,52$ \\
\hline Fibra Óptica G.652.D $(\mathrm{m})$ & 5840 & $\$$ & 4,21 & $\$ 24.586,40$ \\
\hline Fibra Óptica G.652.D $(1000 \mathrm{~m})$ & 6 & $\$$ & 284,00 & $\$$ & $1.704,00$ \\
\hline Fibra Óptica G.657.A $(1000 \mathrm{~m})$ & 10 & $\$$ & 104,00 & $\$$ & $1.040,00$ \\
\hline Conectores OLT & 16 & $\$$ & 1,60 & $\$$ & 25,60 \\
\hline Pigtail ONT & 2048 & $\$$ & 1,60 & $\$$ & $3.276,80$ \\
\hline Manga & 6 & $\$$ & 84,99 & $\$$ & 509,94 \\
\hline $1^{\circ}$ Splitter 1:2 & 32 & $\$$ & 8,99 & $\$$ & 287,68 \\
\hline $2^{\circ}$ Splitter 1:32 & 64 & $\$$ & 32,99 & $\$$ & $2.111,36$ \\
\hline Caja terminal de 4 & 32 & $\$$ & 13,20 & $\$$ & 422,40 \\
\hline Caja terminal de 48 & 64 & $\$$ & 16,20 & $\$$ & $1.036,80$ \\
\hline Roseta Óptica & 2048 & $\$$ & 6,30 & $\$ 12.902,40$ \\
\hline Total & & & & $\$ 178.522,90$ \\
\hline
\end{tabular}

Fuente: Elaboración propia.

\section{Balance de potencia de la red Óptica}

Se realizará el análisis para los casos más críticos de la red que son el usuario más cercano y lejano de cada una de las zonas ya que una vez comprobado que los valores obtenidos en estos casos se garantizara un correcto funcionamiento para los demás casos de la de la res

Para el cálculo del usuario más cercano que se encuentra a 1348m se realizará con las perdidas mininas que se tendrá en la red, donde se utilizará una fibra óptica de tipo monomodo con una longitud de onda de $1550 \mathrm{~nm}$ debido a que es la que menor perdidas presenta por kilómetro y la distancia que recorre la fibra óptica es la más cercana desde la OLT pasando por todos lo demás componentes hasta llegar a la ONT del usuario más cercano.

\section{Tabla 5}

USUARIOS MÁS CERCANO DE CADA UNA DE LAS 6 ZONAS

\begin{tabular}{lccrcrc}
\hline Zonas & \multicolumn{2}{c}{ Distancia } & \multicolumn{2}{c}{ Pérdidas Totales } & \multicolumn{2}{c}{ Potencia Recibida } \\
\hline Zona 1 & 3,54 & $\mathrm{Km}$ & 25,085 & $d B$ & $-18,085$ & $\mathrm{dBm}$ \\
Zona 2 & 1,34 & $\mathrm{Km}$ & 24,530 & $d B$ & $-17,537$ & $\mathrm{dBm}$ \\
Zona 3 & 1,95 & $\mathrm{Km}$ & 24,687 & $d B$ & $-17,687$ & $\mathrm{dBm}$ \\
Zona 4 & 1,48 & $\mathrm{Km}$ & 24,570 & $d B$ & $-17,570$ & $\mathrm{dBm}$ \\
Zona 5 & 3,22 & $\mathrm{Km}$ & 25,005 & $d B$ & $-18,005$ & $\mathrm{dBm}$ \\
Zona 6 & 3,61 & $\mathrm{Km}$ & 25,102 & $d B$ & $-18,102$ & $\mathrm{dBm}$ \\
\hline
\end{tabular}

Fuente: Elaboración propia. 
Para el cálculo del usuario más lejano que se encuentra a 4200m realizará con las perdidas máximas que se tendrá en la red, donde se utilizará una fibra óptica de tipo monomodo con una longitud de onda de $1310 \mathrm{~nm}$ debido a que es la que mayor perdida presenta por kilómetro y la distancia que recorre la fibra óptica es la más alejada desde la OLT pasando por todos lo demás componentes hasta llegar a la ONT del usuario más distante.

Tabla 6

\begin{tabular}{lcccccc}
\multicolumn{6}{c}{ USUARIOS MÁS LEJANOS DE CADA UNA DE LAS 6 ZONAS } \\
\hline \multicolumn{7}{c}{ Pérdidas } \\
Zonas & Distancia & Totales & Potencia Recibida \\
\hline Zona 1 & 4,01 & $\mathrm{Km}$ & 25,6035 & $d B$ & $-22,6035$ & $d B m$ \\
Zona 2 & 1,63 & $\mathrm{Km}$ & 24,7705 & $d B$ & $-21,7705$ & $d B m$ \\
Zona 3 & 3,27 & $\mathrm{Km}$ & 25,3445 & $d B$ & $-22,3445$ & $d B m$ \\
Zona 4 & 2,72 & $\mathrm{Km}$ & 25,1520 & $d B$ & $-22,1520$ & $d B m$ \\
Zona 5 & 4,20 & $\mathrm{Km}$ & 25,7995 & $d B$ & $-22,7995$ & $d B m$ \\
Zona 6 & 4,17 & $\mathrm{Km}$ & 25,8205 & $d B$ & $-22,8205$ & $d B m$ \\
\hline \multicolumn{6}{c}{ Fuente: Elaboración propia. }
\end{tabular}

\section{J. Simulación}

Para el desarrollo de la simulación de la red se trabajará con el software Optisystem de la compañía Optiwave, donde este es de uso gratuitito con 30 días de vigencia desde el momento de su instalación.

Debido al gran número de clientes potenciales se desarrollará la simulación de la red para el usuario más cercano a la OLT que será el primer caso y para el más lejano de la OLT el segundo caso, debido a que si se realiza la simulación de toda la red representaría una sobrecarga datos a procesar para el software y hardware.

Es por eso por lo que serán los dos casos más críticos donde se simulara con perdías mínimas para el primer caso y perdidas máximas para el segundo caso, y así se comprueba el desempeño la red ya que si funciona y se encuentran dentro del estándar para estos dos casos más críticos funcionara correctamente para todos los demás usuarios.

\section{Figura 3}

Diseño de la red GPON simulación

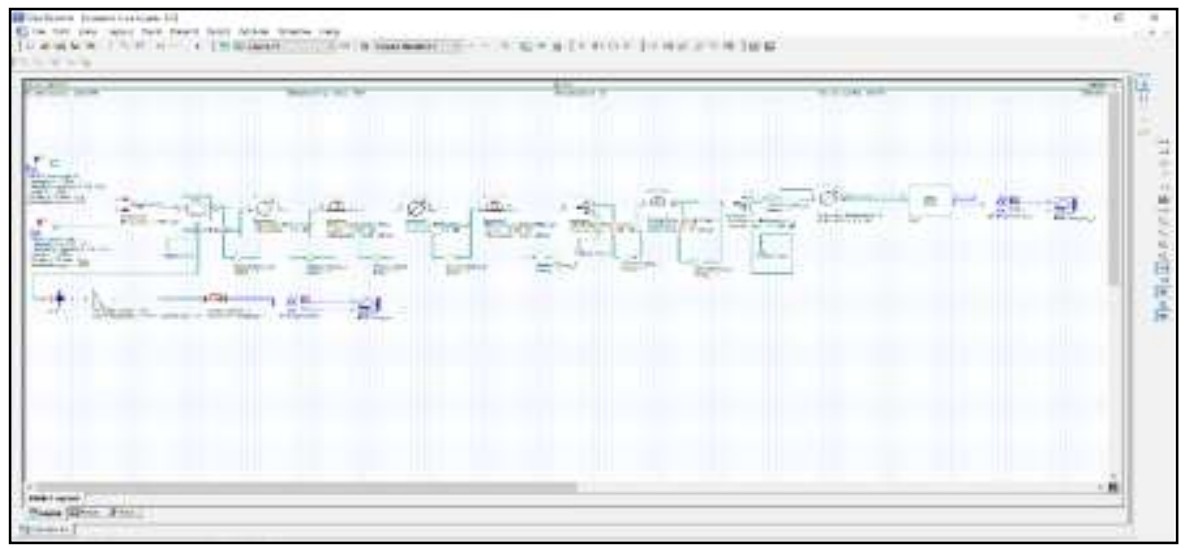

Fuente: Elaboración propia. 
Para la configuración de la simulación del usuario más cercano se debe tomar en cuenta dos factores muy importantes, la OLT que debe proporcionar la potencia máxima y la fibra óptica de tipo monomodo con menores perdidas que es la de longitud de $1550 \mathrm{~nm}$. Como se realizó en el análisis del cálculo de pérdidas y potencia del usuario más cercano.

Configuración de la OLT del usuario más cercano

La OLT está compuesta por un transmisor a la frecuencia de $1550 \mathrm{~nm}$ que representará la señal de video, otro transmisor a la frecuencia de $1490 \mathrm{~nm}$ que será señal de internet, ambos transmisores configurados con una potencia de $7 \mathrm{dBm}$, Un bit rate igual al del sistema 2.5Gbps y una modulación NRZ. Las dos señales se juntan en el multiplexor por división de longitud de onda WDM Add para obtener una única señal a trasmitir, esta ingresa al Circulator Bidirectional que se encarga de obtener el canal downstream y upstream, un Attenuador Bidireccional que representa las pérdidas de conectores y fusiones en cada etapa de la red.

\section{Figura 3}

\section{OLT del primer caso más cercano}

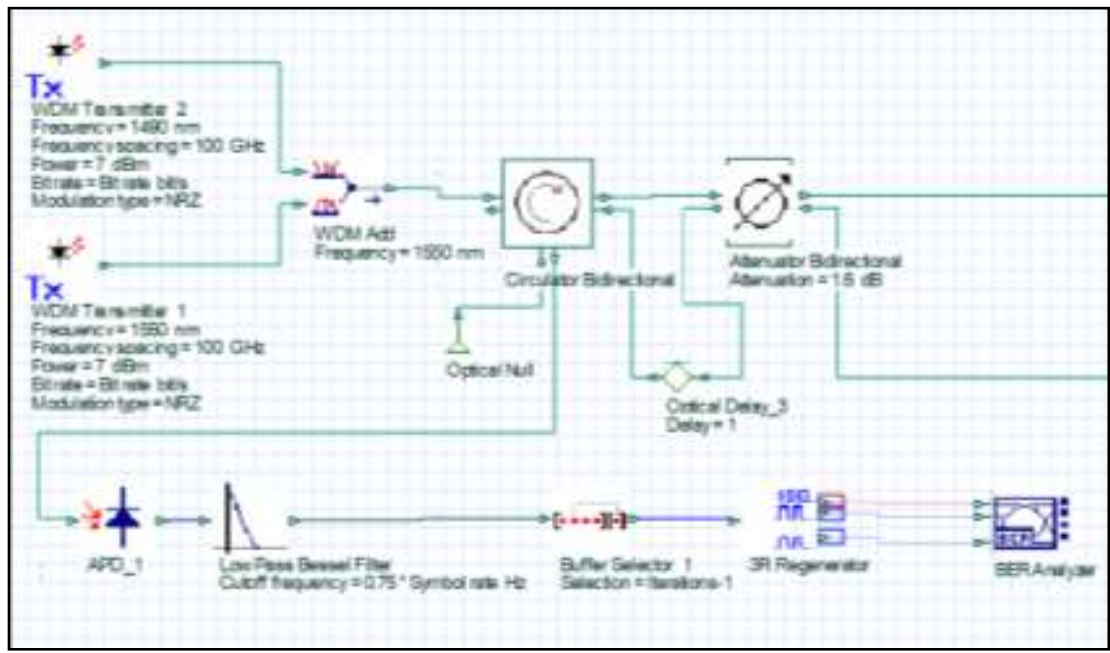

Fuente: Elaboración propia.

La red ODN está compuesta por: Bidirectional Optical Fiber, Attenuator Bidireccional, dos Splitter Bidirectional de 1:2 y de 1:32 el software por defecto da una pérdida de 3.011 $\mathrm{dBm}$ es por eso por lo que se inserta manualmente pérdidas de $0.489 \mathrm{dBm}$ y $14.489 \mathrm{dBm}$ respectivamente para alcanazar las perdidas reales de los splitter. Con una distancia total de $1.34 \mathrm{~km}$.

\section{Figura 4}

ODN del primer caso de red GPON

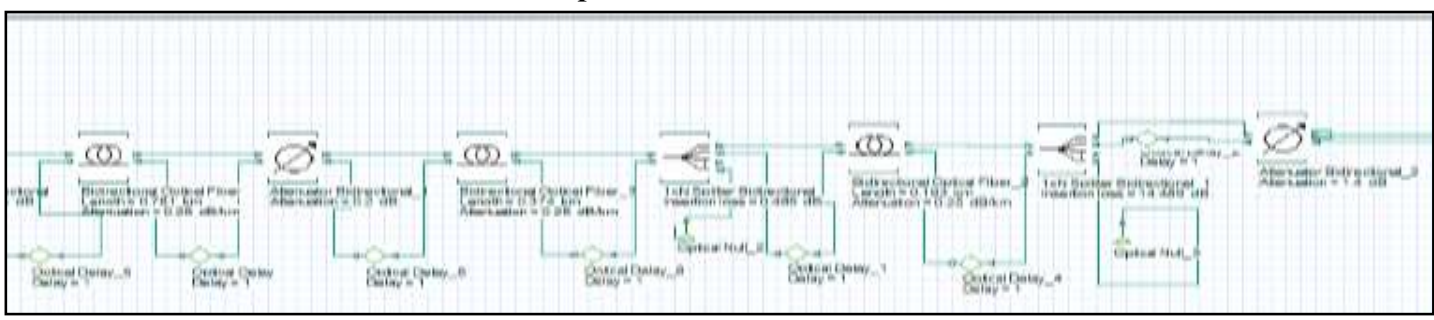

Fuente: Elaboración propia. 
Para la configuración de la simulación del usuario más lejano se debe tomar en cuenta dos factores muy importantes, la OLT que del usuario más lejano debe proporcionar la potencia mínima y la fibra óptica de tipo monomodo con la característica de perdidas mayores que esta posee. Como se realizó en el análisis del cálculo de pérdidas y potencia del usuario más lejano.

Configuración de la OLT del usuario más lejano

La OLT va a tener la misma configuración que en el usuario más cercano de la zona 2 ahora lo que va a diferenciar es la potencia de transición para simular la red con pérdidas máximas, esta potencia va a ser ahora de $3 \mathrm{dBm}$.

\section{Figura 5}

\section{OLT del segundo caso más lejano}

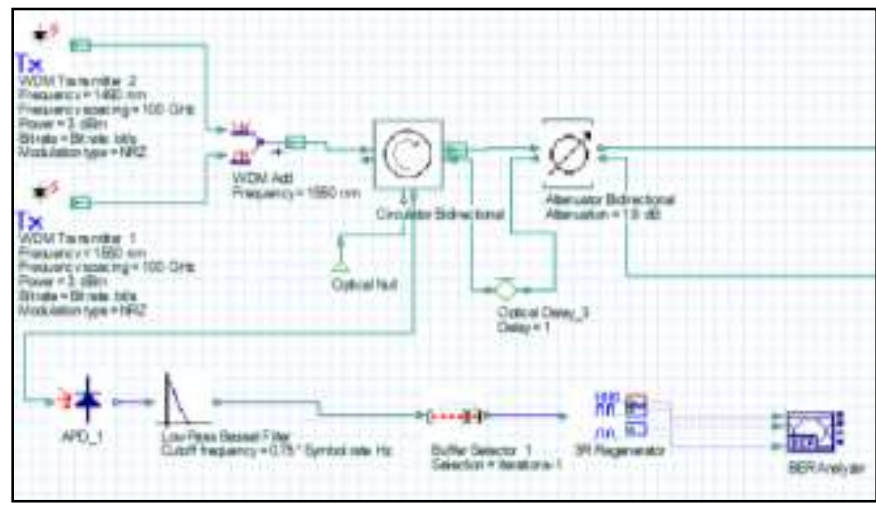

Fuente: Elaboración propia.

La red ODN está compuesta por: Bidirectional Optical Fiber, Attenuator Bidireccional, dos Splitter Bidirectional de 1:2 y de 1:32 el software por defecto da una pérdida de 3.011 $\mathrm{dBm}$ es por eso por lo que se inserta manualmente pérdidas de $0.489 \mathrm{dBm}$ y $14.489 \mathrm{dBm}$ respectivamente para alcanazar las perdidas reales de los splitter. Con una distancia total de $4.20 \mathrm{~km}$.

\section{Figura 6}

ODN del segundo caso más lejano

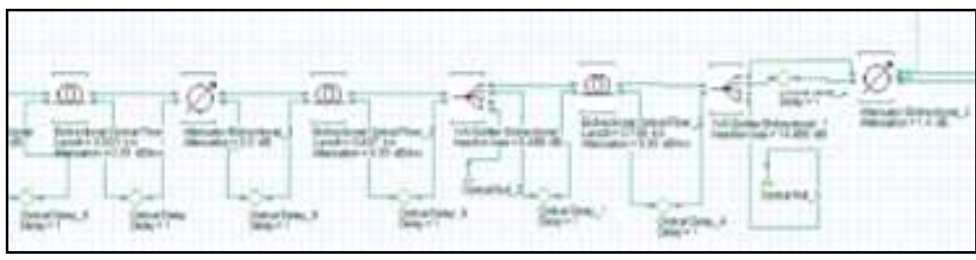

Fuente: Elaboración propia.

\section{Configuración de la ONT de la Red GPON}

La ONT está conformado por un subsistema y un 3R Generator que es el encargado de reconstruir la señal para observar los resultados obtenidos en el BER Analyzar.

El subsistema de la ONT está conformado por un Pin APD que detecta la señal óptica y la transforma en eléctrica es por eso por lo que el color de línea de conexión cambia pasa 
por un Low Pass Bessel Filter encargado de separar las señales de video e internet y eliminar el ruido introducido del sistema.

También consta de una etapa de trasmisión que representa el canal de subida de internet donde se utiliza WDM Transmiter con una potencia de $5 \mathrm{dBm}$ suministrada por el fabricante del equipo ONT, la longitud de onda a $1310 \mathrm{~nm}$. Para simular el acceso múltiple por división de tiempo (TDMA) se emplearon Dynamic Y Select con un intervalo de tiempo que se le asigna a cada cliente final para que los datos puedan ser trasmitidos por el canal upstream hacia la OLT.

\section{Figura 7}

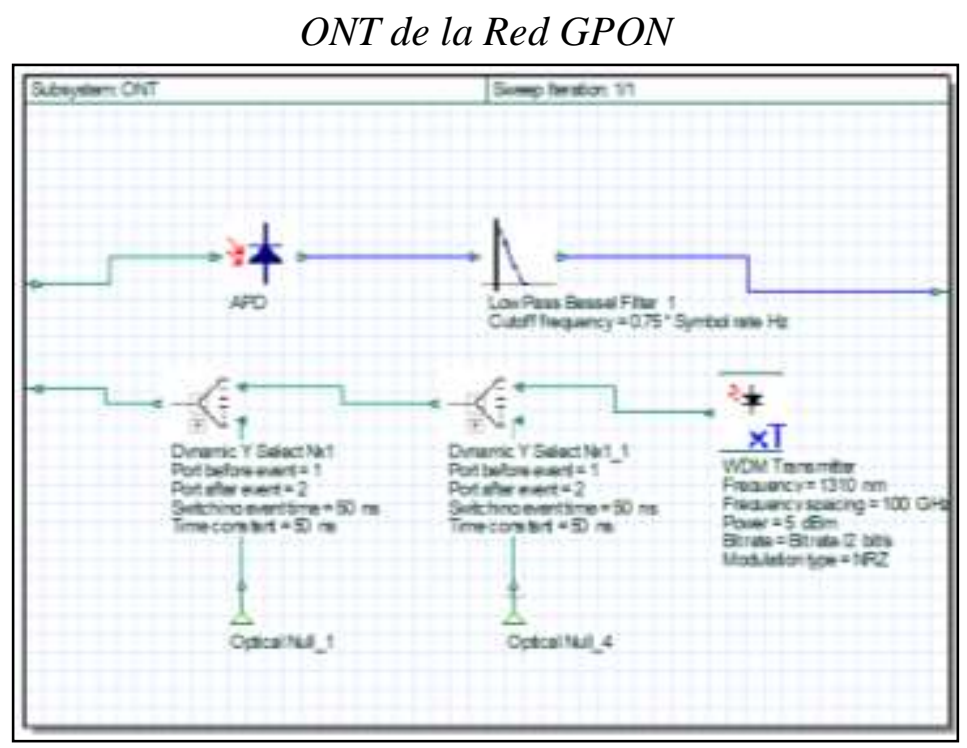

Fuente: Elaboración propia.

\section{Resultados}

En el presente capitulo se realizará un análisis de los datos obtenidos en las encuestas, los resultados de las pruebas hechas en la red comparando los valores de obtenido teóricamente con los valores conseguidos en la simulación de los casos críticos de cada una de las 6 zonas de la red de fibra óptica.

\section{K. Análisis del balance de potencias de los casos críticos}

Primer caso critico usuario más cercano, se analizó el balance de potencias de cada uno de los usuarios más críticos de las 6 zonas, es decir cuál es el usuario más cercano.

Para el primer caso crítico del usuario más cercano es importante detallar que lo que deseamos analizar es que se obtenga la cantidad de perdidas mínimas que hay en el enlace, ya que si un caso fortuito la potencia del trasmisor aumentara por encima del promedio que trabaja en condiciones normales que es en el rango de 3 a $7 \mathrm{dBm}$. Este aumento no afecte a los equipos receptores es por eso por lo que el balance de potencia se realiza con la potencia máxima que puede suministrar el equipo transmisor de $7 \mathrm{dBm}$. 
Tabla 6

ATENUACION RECIBIDA DEL PRIIMER CASO

\begin{tabular}{cccrcrc}
\hline Zona & Distancia & & Perdidas & Potencia recibida & \\
\hline Zona 1 & 3,54 & $K m$ & 25,085 & $d B$ & $-18,085$ & $d B m$ \\
Zona 2 & 1,34 & $K m$ & 24,530 & $d B$ & $-17,537$ & $d B m$ \\
Zona 3 & 1,95 & $K m$ & 24,688 & $d B$ & $-17,688$ & $d B m$ \\
Zona 4 & 1,48 & $K m$ & 24,570 & $d B$ & $-17,570$ & $d B m$ \\
Zona 5 & 3,22 & $K m$ & 25,005 & $d B$ & $-18,005$ & $d B m$ \\
Zona 6 & 3,61 & $K m$ & 25,103 & $d B$ & $-18,103$ & $d B m$ \\
\hline
\end{tabular}

Fuente: Elaboración propia.

Se observa en la tabla 6 que la atenuación recibida de los usuarios más cercano de cada una de las 6 zonas, con potencia máxima suministrada por el equipo transmisor se encuentra dentro de la recomendación del estándar es decir debe estar por encima de los $17 \mathrm{~dB}$ y no debe sobrepasar los $32 \mathrm{~dB}$, manteniendo el marguen de seguridad de $3 \mathrm{~dB}$.

Comprobamos que cada uno de los valores obtenidos se encuentra dentro del rango de funcionamiento garantizando un correcto funcionamiento de la red.

Para el segundo caso crítico del usuario más lejano, Se analizo el balance de potencias de cada uno de los usuarios más críticos de las 6 zonas, es decir cuál es el usuario más lejano.

En el segundo caso crítico del usuario más lejano es importante detallar que lo que deseamos analizar es que se obtenga la cantidad de perdidas máximas que hay en el enlace, ya que si un caso fortuito la potencia del trasmisor disminuyera por debajo del promedio que trabaja en condiciones normales que es en el rango de 3 a $7 \mathrm{dBm}$. Esta disminución no afecte a los equipos receptores es por eso por lo que el balance de potencia se realiza con la potencia mínima que puede suministrar el equipo transmisor de $3 \mathrm{dBm}$.

Tabla 7

ATENUACION RECIBIDA DEL SEGUNDO CASO

\begin{tabular}{lrlrlrl}
\hline Zona & Distancia & & Perdidas & & Potencia recibida & \\
\hline Zona 1 & 4,01 & $\mathrm{Km}$ & 25,604 & $d B$ & $-22,604$ & $\mathrm{dBm}$ \\
Zona 2 & 1,63 & $\mathrm{Km}$ & 24,771 & $d B$ & $-21,771$ & $\mathrm{dBm}$ \\
Zona 3 & 3,27 & $\mathrm{Km}$ & 25,345 & $\mathrm{~dB}$ & $-22,345$ & $\mathrm{dBm}$ \\
Zona 4 & 2,72 & $\mathrm{Km}$ & 25,152 & $d B$ & $-22,152$ & $\mathrm{dBm}$ \\
Zona 5 & 4,20 & $\mathrm{Km}$ & 25,670 & $\mathrm{~dB}$ & $-22,670$ & $\mathrm{dBm}$ \\
Zona 6 & 4,17 & $\mathrm{Km}$ & 25,660 & $\mathrm{~dB}$ & $-22,957$ & $\mathrm{dBm}$ \\
\hline
\end{tabular}

Fuente: Elaboración propia.

Se observa que la atenuación recibida de los usuarios más lejano de cada una de las 6 zonas, con potencia mínima suministrada por el equipo transmisor se encuentra dentro de la recomendación del estándar es decir debe estar por encima de los 17 dB y no debe sobrepasar los $32 \mathrm{~dB}$, manteniendo el marguen de seguridad de $3 \mathrm{~dB}$.

Comprobamos que cada uno de los valores obtenidos se encuentra dentro del rango de funcionamiento garantizando un correcto funcionamiento de la red 
Análisis de los valores obtenidos en simulación

Se procederá a analizar cada uno los valores de atenuación y los diagramas de ojo que se obtiene como resultado del software OptiSystem que contiene los valores BER y factor Q.

\section{Figura 8}

\section{Diagrama de ojo ideal}

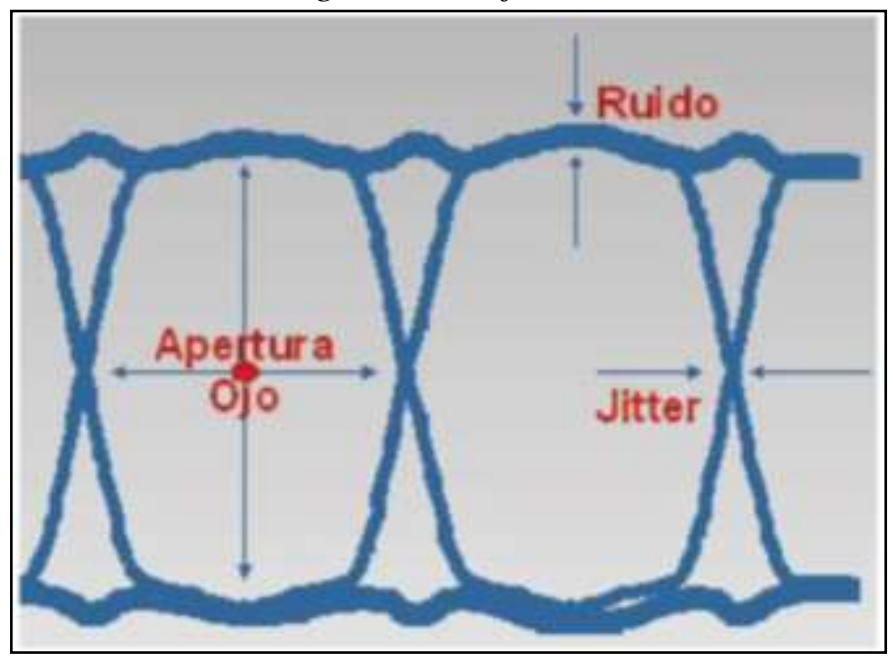

Fuente: Rivera and Leandro(2018)

Se utilizará como referencia la figura 1-3 de un diagrama de ojo ideal con una apertura amplia y poco ruido en los bordes del ojo para comparar con los diagramas de ojo de los casos críticos y saber si se acercan a aun diagrama ideal.

En los resultados de los casos más críticos obtenidos en las diferentes ONT de cada una de las zonas se verificara que estos se encuentren dentro de los parámetros que establecidos por la normativa (ITU G.984.2, 2019) donde el factor Q que es acerca de la calidad de la conexión, debe tener un valor igual a 6 o mayor y el BER debe ser igual a $1^{*} 10^{\wedge}(-10)$ o menor, esto quiere decir que se puede generar un bit errado por cada 10 mil millones de bits que se trasmitieron por la OLT.

A continuación, se realizará el análisis paso a paso del usuario más crítico de toda la red que ese encuentra en la zona 2, ya que para los demás casos de las demás zonas se analizará de manera similar.

Los resultados del usuario más cercano de la zona 2 están representados en el diagrama de ojo obtenido de la simulación de la red del primer caso donde se representa de una manera visual el factor Q y el BER combinados en este diagrama, es decir muestra una posición aleatoria de combinaciones de bits unos y ceros que fueron transmitidos por la OLT. 
Figura 9

\section{Diagrama de ojo del primer caso}

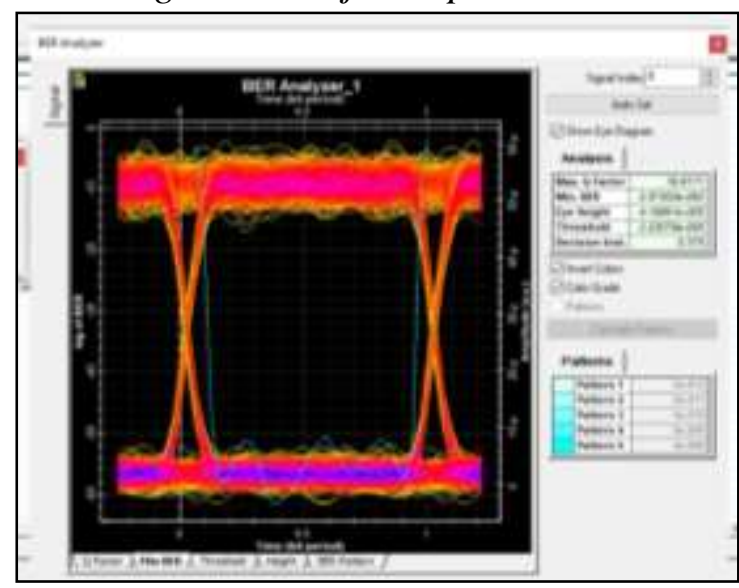

Fuente: Elaboración propia.

En el primer caso del usuario más cercano de la zona 2 se obtiene como resultado que el diagrama del ojo tiene una abertura amplia y un ruido aceptable haciendo una comparación con la figura 1-3 se tiene un diagrama de ojo que se acerca al ideal y los valores obtenidos lo demuestra, donde su factor Q 16.6171 y su BER de $2.57303^{*} 10^{\wedge}(-$ 62) comprobando que los valores se encuentran dentro de los parámetros de la normativa.

\section{Figura 10}

Atenuación del primer caso

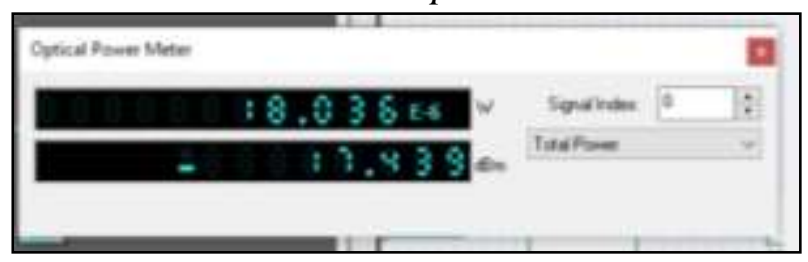

Fuente: Elaboración propia.

Ahora se va a analizo la atenuación obtenida de la simulación con pérdidas mínimas del primer caso es decir la potencia máxima de la OLT y la menor cantidad de pérdidas presentes en el enlace y compararla con la potencia de recepción que se calculó en la sección 2.15.2 donde se obtuvo una atenuación de -17.5.37 dBm

En la simulación se obtuvo una atenuación de -17.439 dbm que se acerca bastante a la calculada solo con un $0.09 \mathrm{dbm}$ de diferencia donde se comprueba que los valores conseguidos se encuentran por encima del valor que recomienda el estándar (ITU G.984.2, 2019) clase $\mathrm{C}+$ que es de $-17.00 \mathrm{dbm}$ conde con este valor se garantiza un correcto funcionamiento de la red en el primer caso más crítico de la zona 2.

Los resultados del usuario más lejano que se encuentra en la zona 5 están representados en el diagrama de ojo obtenido de la simulación de la red del segundo caso, donde se representa de una manera visual el factor Q y el BER combinados en este diagrama, es decir muestra una posición aleatoria de combinaciones de bits unos y ceros que fueron transmitidos por la OLT. 
Figura 11

Diagrama del ojo del segundo caso

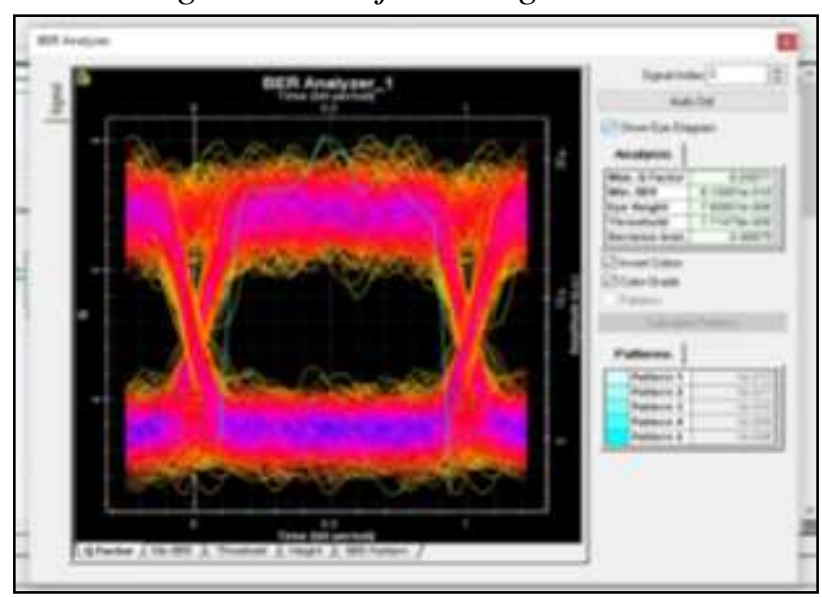

Fuente: Elaboración propia.

En el segundo caso del usuario más lejano se obtiene como resultado que el diagrama del ojo tiene una abertura considerable y mucho ruido pero es aceptable ya que estamos evaluando a la red en un caso extremo haciendo una comparación con la figura 1-3 se tiene que el diagrama de ojo que aún conserva su forma original pese al ruido presente y la calidad de la señal en comparación con el ideal y los valores obtenidos lo demuestra donde su factor Q es mayor a 6 6.03071y un BER de 8..13001*10^(-10) comprobando que los valores se encuentran dentro de los parámetros de la normativa.

Figura 12

Atenuación del segundo caso

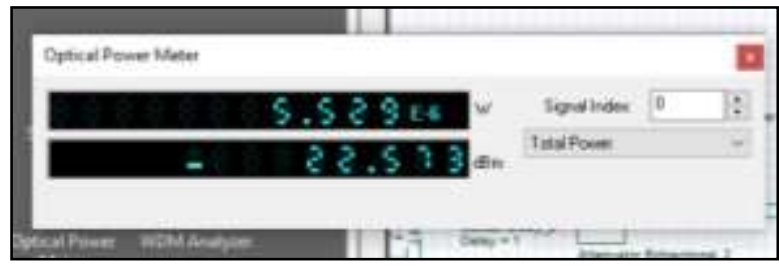

Fuente: Elaboración propia.

Ahora se ha analizo la atenuación obtenida de la simulación con pérdidas máximas del segundo caso es decir la potencia mínima de la OLT y la mayor cantidad de pérdidas del enlace y compararla con la potencia de recepción que se calculó en la sección 2.15.5 donde se obtuvo una atenuación de -22.67 dbm

En la simulación se obtuvo una atenuación de $-22.573 \mathrm{dbm}$ que se acerca bastante a la calculada solo con un $0.09 \mathrm{dbm}$ de diferencia donde se comprueba que los valores conseguidos se encuentran por debajo del valor que recomienda el estándar (ITU G.984.2, 2019) clase $\mathrm{C}+$ que es de $-32.00 \mathrm{dbm}$ donde con este valor se garantiza un correcto funcionamiento de la red en el segundo caso más crítico de la zona 5. Por ende una vez comprobados los valores de los dos casos más críticos se garantizar un correcto funcionamiento de la red para todos los demás casos.

Resultados de los casos críticos de las 6 zonas 
A continuación, se muestra todos los valores obtenidos de la simulación de todos los casos críticos de cada una de las 6 zonas. Se tiene como primer dato una comparación de la atenuación obtenida con la atenuación simulada donde los valores obtenidos son casi iguales tan solo con un margen de error del $0.5 \%$ aproximadamente ya que la atenuación obtenida con la simulada existe una diferencia de $0.09 \mathrm{dBm}$ en promedio.

Ahora comparando estos datos con la recomendación ITU G.984.2 clase C + se observar que para todos los casos críticos lejano y cercano de las 6 zonas están dentro del rango, mayor a $-17 \mathrm{dBm}$ y menor a $-32 \mathrm{dBm}$ manteniendo el margen de seguridad de $6 \mathrm{~dB}$ en todos los casos.

\section{Tabla 8}

RESULTADOS OBTENIDOS DE CADA UNA DE LAS ZONAS

\begin{tabular}{|c|c|c|c|c|c|}
\hline \multicolumn{3}{|c|}{ Usuario más cercano } & \multicolumn{3}{|c|}{ Usuario más lejano } \\
\hline Zonas & Distancia & Atenuación Obtenida & Atenuación Simulada & Factor $\mathrm{Q}$ & BER \\
\hline \multirow[t]{2}{*}{ Zona 1} & 3,54 Km & $-18,085 \mathrm{dBm}$ & $-17,988 \mathrm{dBm}$ & 14,8238 & $5,0740 e-50$ \\
\hline & $4,01 \mathrm{Km}$ & $-22,604 \mathrm{dBm}$ & $-22,507 \mathrm{dBm}$ & 6,0236 & $8,5209 e-10$ \\
\hline \multirow[t]{2}{*}{ Zona 2} & $1,34 \mathrm{Km}$ & $-17,537 \mathrm{dBm}$ & $-17,439 \mathrm{dBm}$ & 16,6171 & $2,5730 \mathrm{e}-62$ \\
\hline & $1,63 \mathrm{Km}$ & $-21,771 \mathrm{dBm}$ & $-21,674 \mathrm{dBm}$ & 7,2299 & $2,4119 e-13$ \\
\hline \multirow[t]{2}{*}{ Zona 3} & $1,95 \mathrm{Km}$ & $-17,688 \mathrm{dBm}$ & $-17,591 \mathrm{dBm}$ & 16.0891 & $1,4969 e-58$ \\
\hline & $3,27 \mathrm{Km}$ & $-22,345 \mathrm{dBm}$ & $-22,248 \mathrm{dBm}$ & 6,4133 & $7,1035 e-11$ \\
\hline \multirow[t]{2}{*}{ Zona 4} & $1,48 \mathrm{Km}$ & $-17,570 \mathrm{dBm}$ & $-17,474 \mathrm{dBm}$ & 16,5516 & $7,6557 \mathrm{e}-62$ \\
\hline & $2,72 \mathrm{Km}$ & $-22,152 \mathrm{dBm}$ & $-22,055 \mathrm{dBm}$ & 6,5583 & $2,7160 \mathrm{e}-11$ \\
\hline \multirow[t]{2}{*}{ Zona 5} & 3,22 Km & $-18,005 \mathrm{dBm}$ & $-17,954 \mathrm{dBm}$ & 14,6466 & $6,9210 e-49$ \\
\hline & $4,20 \mathrm{Km}$ & $-22,670 \mathrm{dBm}$ & $-22,573 \mathrm{dBm}$ & 6,0307 & $8,1300 \mathrm{e} 10$ \\
\hline \multirow[t]{2}{*}{ Zona 6} & 3,61 Km & $-18,103 \mathrm{dBm}$ & $-18,006 \mathrm{dBm}$ & 15,0417 & $1,9320 \mathrm{e}-51$ \\
\hline & $4,17 \mathrm{Km}$ & $-22,690 \mathrm{dBm}$ & $-22.563 \mathrm{dBm}$ & 6,0439 & $7,4902 \mathrm{e} 10$ \\
\hline
\end{tabular}

Fuente: Elaboración propia.

Ahora revisando los valores de factor Q todos los datos son mayor a 6, en los usuarios más cercanos en mucho mayor llegando hasta 16 donde se tienen una calidad de conexión excelente a diferencia de los usuarios más lejanos que no es excelente pero es aceptable. De igual manera analizo los valores de BER que se encuentran muy por debajo de $1 * 10 *$ e10 que es excelente ya que no se pierde casi nada de bits en los usuarios más cercanos a diferencia de los lejano que no es tan bajo se pierde menos de un bit por cada mil millones de bits transmitidos es aceptable.

Cabe recalcar que estos son casos críticos es decir con condiciones extremas que se puedan presentar en la red potencia mínima suministrada por la OLT y la mayor cantidad de pérdidas, con todas estas condiciones se mantienen por encima del valor de la recomendación que es 6 para el factor Q y por debajo $1 * 10 \mathrm{E}-10$ con respecto al BER.

Es por esos que los valores se diferencian bastante del usuario más cercano del usuario más lejano ya el primero es con máxima que potencia y segundo es con potencia mínima. 


\section{Conclusiones}

- Se realizó el estudio de la red actual de la parroquia Huambaló donde en base a las encuestas se obtuvo como resultado que su red de acceso actual para los usuarios finales para servicio de internet es el medio guiado de cobre y el de video mediante televisión satelital, evidenciándose la necesidad de una migración de tecnología hacia fibra óptica de tipo monomodo que permita tener mejores beneficios como: velocidades de transmisión más altas, una menor latencia entre otras.

- Se analizo los estándares vigentes por la unión internacional de telecomunicaciones recomendación ITU-T G984.x, donde se eligió la tecnología GPON como la más apropiada por sus características principales: capacidad, niveles de splitter y distancia en kilómetros, siendo la normativa adoptada la recomendación ITU G.984.2 clase C+, ya que esta es la más optima que se empleó para el diseño de la red, debido a su un margen mayor de sensibilidad mínima y máxima en la OLT como en la ONT con respectos con las demás clases, teniendo así un amplio catálogo de equipos que se ofertan en el mercado y cumplen con estas características al momento que sea implementada el diseño de red en la parroquia Huambaló.

- Se diseño una red convergente duoplay con la integración de los servicios de video e internet que serán provistos por la empresa American Cable S.A. en base a los planos de infraestructura predial y acceso vial de la parroquia Huambaló, que se acoplaron a las necesidades y requerimientos de cada uno de los posibles clientes finales, donde a la parroquia se dividió en 6 zonas específicas las cuales se fundamente especialmente en su concentración demográfica como comercial, ubicando a la OLT en el parte central de las zonas de la cual saldrán las redes feeder, de distribución y dispersión, obteniendo una mejor distribución de usuarios y control de los mismo.

- Mediante la utilización del software de OptiSystem se evaluó la red de acceso GPON para los dos casos más críticos, donde se obtuvo los siguientes datos, en el primer caso que corresponde al usuario más cercano que se encuentra en la zona 2 se simulo con la menor cantidad de pérdidas que se podría presentar y potencia máxima del equipo de transmisión OLT, donde se obtuvo los siguientes resultados: en atenuación $-17.439 \mathrm{dbm}$, factor Q 16.6171 y un BER de $2.57303 * 10^{\wedge}(-62)$ y para en el segundo caso que corresponde al usuario más lejano que se encuentra en la zona 5, se simulo con la mayor cantidad de pérdidas que se podría presentar y potencia al mínimo del equipo de transmisión OLT, donde se obtuvo los siguientes resultados: en atenuación $-22.573 \mathrm{dbm}$, factor Q 6.03071 y un BER de $8.13001 * 10^{\wedge}(-10)$ comprobando que los valores se encuentran dentro de los parámetros de la normativa ITU G.984.2 Clases C+ afianzando así un correcto funcionamiento de la red para todos los demás usuarios. 


\section{Referencias bibliográficas.}

Caizaguano criollo, Santiago Luis. (2020) Diseño de una red convergente de fibra óptica para interconectar los Campus de la Universidad de la Américas, 9-10. Recuperado desde: http://repositorio.puce.edu.ec:80/xmlui/handle/2200 0/8078

Díaz Ataucuri, Daniel, Sifuentes, Isabel Guadalupe, Chamorro, Rudy, Tucto, Tucto \& Curioso, Walter H. (2014). Despliegue de infraestructura de fibra óptica para formar la Red Nacional de Investigación y Educación en el Perú. 1617. Recuperado desde: https://docplayer.es/2343221-Despliegue-de-infraestructurade-fibra-optica-para-formar-la-red-nacional-de-investigacion-y-educacion-en-elperu.html

Huawei MA5680T. (2020) Price and Datasheet Recuperado desde: https://www.thunderlink.com/HUAWEI-MA5680T_p575.html

ITU G.984.2, G.984.2. G.984.2: (2019) Redes ópticas pasivas con capacidad de gigabits 22-26. Recuperado desde: https://www.itu.int/rec/T-REC-G.984.2-201908-I/es

Joskowicz, José. (2013). Breve historia de las telecomunicaciones. Instituto de Ingenieria Eléctrica de la republica de Uruguay. 43-46. Recuperado desde: http://iie.fing.edu.uy/ense/asign/ccu/material/docs/Historia\%20de\%201as\%20Tel ecomunicaciones.pdf

OPTIWAVE SYSTEMS INC. (2020). OptiSystem Overview. Dans: Optiwave. Recuperado desde: https://optiwave.com/optisystem-overview/

Pavón, Cristian Marcelo \& Gadpr Huambaló. (2015). Plan de desarrollo y ordenamiento territorial de la parroquia rural Huambaló. Octobre 2015, p. 245. Recuperado desde:

http://app.sni.gob.ec/snilink/sni/PORTAL_SNI/data_sigad_plus/sigadplusdocum entofinal/1865

015860001_PDOT_HUAMBALO_OCTUBRE_2015_FINAL_30-10-2015_2121-19.pdf

Rivera Velasco, Leandro Bruno. (2018). Diseño y simulación de una red GPON para ofrecer el servicio de Triple Play en el sector de San Antonio de Ibarra para la CNT-EP. Recuperado desde: http://bibdigital.epn.edu.ec/handle/15000/19291.

TELNET, Garcia. (2014). Soluciones y casos de éxito. Dans: TELNET Redes Inteligentes Recuperado desde: https://www.telnet-ri.es/soluciones-y-casos-de-exito/

ZTE C300 (2020). Price and Datasheet Recuperado desde: https://www.thunderlink.com/gtgh-c-plus2.html

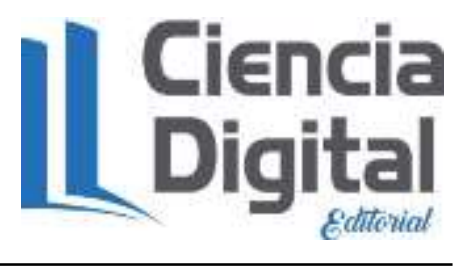




\section{PARA CITAR EL ARTÍCULO INDEXADO.}

Martínez Guashima, O., Parra Mayorga, O. S., \& Peñafiel Ortega, I. A. (2021). Diseño de una Red de Acceso de Fibra Óptica para Proveer Video e Internet y Evaluación de parámetros de calidad sobre la red caso de estudio "Sector de Huambaló" . AlfaPublicaciones, 3(3.1), 216-237. https://doi.org/10.33262/ap.v3i3.1.88

\section{Ciencia \\ LDigital}

El artículo que se publica es de exclusiva responsabilidad de los autores y no necesariamente reflejan el pensamiento de la Revista Alfa Publicaciones.

El artículo queda en propiedad de la revista y, por tanto, su publicación parcial y/o total en otro medio tiene que ser autorizado por el director de la Revista Alfa Publicaciones.
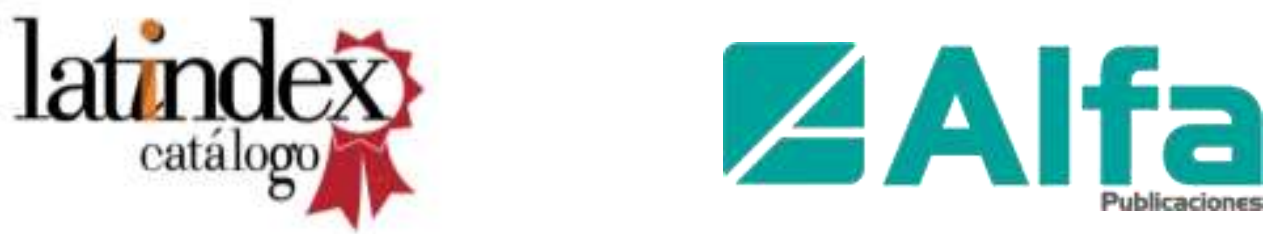